\title{
Fault Diagnosis and Fault Tolerant Control for Non-Gaussian Singular Time-Delayed Stochastic Distribution Systems with Disturbance Based on the Rational Square-Root Model
}

\author{
Yuancheng Sun and Zhanhong Liang \\ School of Electrical Engineering, Zhengzhou University, Zhengzhou, Henan 450001, China \\ Correspondence should be addressed to Zhanhong Liang; liangzhanhong@zzu.edu.cn
}

Received 23 March 2016; Revised 14 July 2016; Accepted 28 July 2016

Academic Editor: Manuel Pineda-Sanchez

Copyright (C) 2016 Y. Sun and Z. Liang. This is an open access article distributed under the Creative Commons Attribution License, which permits unrestricted use, distribution, and reproduction in any medium, provided the original work is properly cited.

For the non-Gaussian singular time-delayed stochastic distribution control (SDC) system with unknown external disturbance where the output probability density function (PDF) is approximated by the rational square-root B-spline basis function, a robust fault diagnosis and fault tolerant control algorithm is presented. A full-order observer is constructed to estimate the exogenous disturbance and an adaptive observer is used to estimate the fault size. A fault tolerant tracking controller is designed using the feedback of distribution tracking error, fault, and disturbance estimation to let the postfault output PDF still track desired distribution. Finally, a simulation example is included to illustrate the effectiveness of the proposed algorithms and encouraging results have been obtained.

\section{Introduction}

In order to improve the reliability of practical stochastic systems, fault diagnosis and fault tolerant control for stochastic dynamic systems has long been one of the important areas of control theory and application [1-4]. Stochastic distribution control (SDC) system is a new branch of stochastic system control in which the output is the non-Gaussian probability density function (PDF) of the system output. The equations of these systems describe the relationship between the input PDF and output PDF of systems rather than the traditional relationship between input and output. SDC theory was proposed by professor Wang [5], which has been applied on some actual processes, for instance, the paper evenness control in the process of paper making, high polymer polymerization process of chemical industry, and flame distribution control. In the framework of nonGaussian SDC systems, it has very important theoretical significances and deep application prospect to develop fault diagnosis and fault tolerant control technology for complex industrial processes in which the control product quality and the distribution of indirect indicators need to be controlled.
With the study of fault diagnosis for non-Gaussian SDC systems, some fault diagnosis algorithms have been proposed. For fault diagnosis of SDC systems, observer or filter-based methods are mainly used so far [6-10], in which the information of system output PDF and other measured information generate residuals in order to analyze and estimate the change of fault. A stable filter-based residual generator is constructed such that the fault can be detected and diagnosed for general stochastic systems in [6]. In [7], a nonlinear neural network observer is designed for fault diagnosis in which the adaptive tuning rule for network parameters is determined by the Lyapunov stability theorem. In [8] a fault diagnosis algorithm is proposed based on iterative learning observer for SDC system. Otherwise, in [11], a novel fault-estimation observer is designed for Takagi-Sugeno (T-S) fuzzy systems with actuator faults, and the problem of fault tolerant control is addressed. For fault tolerant control, the active fault tolerant control is mainly used so far. Combined with the controller design method without fault, such as optimal control, PI control, sliding mode control, and model reference adaptive control, the controller can be reconfigured or reconstructed when 
fault occurred. When the target PDF is known, the purpose of fault tolerant control is to make the output PDF of the systems still track a given PDF as close as possible after the fault happened. It has been shown in [8] that an optimal control strategy is designed to reconfigure the controller to compensate the influence of the fault on the system performance. A fault tolerant controller based on PI tracking control is proposed for a time-delayed SDC system in [12] and for singular time-delayed SDC system in [13]. In [14], a fuzzy fault tolerant control scheme is developed to guarantee the closed-loop system to be exponentially stable in mean square. When the target PDF is unknown, introduce the concept of entropy to fault tolerant control of non-Gaussian SDC systems, in which the purpose is to minimize the uncertainty of the system output after fault happened $[15,16]$.

Although the model descriptions of SDC systems are always the dynamic link relations between the input and the weights of output PDFs, the conventional dynamic links do not meet the practical requirements because of the existence of some algebraic constraint conditions between some state variables. Thus, it is necessary to study the SDC systems in the framework of singular systems $[8,13,16-18]$. Due to the material delivering by conveyor belt, system modeling, and data operation and transmission, time-delay widely exists in actual industrial systems. Time-delay can make system performance degradation and even make systems unstable; at the same time, time-delay will largely reduce the effectiveness of the fault diagnosis and fault tolerant control. The results of fault diagnosis and fault tolerant control for singular timedelayed SDC systems are focused on the model approximated by the linear or square-root B-spline model $[13,18]$. The rational square-root $\mathrm{B}$-spline model can guarantee that the weights of feedback control are positive at the same time independent of each other compared with other B-spline models [19]. External disturbance widely exists in various industrial processes and this situation will turn to be very complicated for fault diagnosis $[9,20]$, but controller design to eliminate the influence of disturbance is not considered. Thus, it is significant to study fault diagnosis and fault tolerant control for non-Gaussian singular time-delayed SDC system with external disturbance based on the rational square-root model approximation.

In this paper, a robust fault diagnosis and fault tolerant control approach is proposed for non-Gaussian singular time-delayed stochastic distribution system with external disturbance based on the rational square-root approximation. The external disturbance is considered and supposed to be generated by a linear exogenous system, and a full-order observer is designed to estimate the disturbance. Then an adaptive observer is constructed to estimate fault information. The gain matrices can be determined by solving the corresponding linear matrix inequalities (LMIs). In order to eliminate the influence of fault and disturbance, a fault tolerant tracking controller is designed to track the desired PDF. An augmentation control input is defined to contain the feedback of output PDF tracking error and the estimation of fault and disturbance, so that the control input of the SDC system can eliminate the influence of fault and disturbance on the system performance, leading to fault tolerant tracking control. Finally, the computer simulation results show the effectiveness of the algorithm.

The main contributions of this paper can be summarized as follows. (1) Comparing with most of the existing studies of SDC system with disturbance, integrated fault diagnosis and fault tolerant control is achieved; rather only fault diagnosis is considered. Besides, the influence of disturbance to the system performance can be rejected by the fault tolerant controller. (2) The rational square-root model is used to approximate the output PDF of the singular time-delayed SDC system, as a contrast, linear, or square-root model is used in the existing studies of singular time-delayed SDC system.

\section{Model Description}

Denoting $\gamma(y, u(t))$ as the PDF of the system output with $y$ being defined on a known bounded interval $[a, b]$, the continuous singular time-delayed SDC system can be expressed as follows:

$$
\begin{aligned}
E \dot{x}(t)= & A x(t)+A_{d} x(t-\tau)+B u(t)+N F(t) \\
& +B_{d} d(t) \\
V(t)= & D x(t) \\
x(t)= & \varphi(t), \quad t \in[-\delta, 0] \\
\sqrt{\gamma(y, u(t))}= & \frac{C(y) V(t)}{\sqrt{V^{T}(t) \Sigma_{1} V(t)}} \\
\Sigma_{1}= & \int_{a}^{b} C^{T}(y) C(y) d y
\end{aligned}
$$

where $x(t) \in R^{n}$ is the state vector, $u(t) \in R^{m}$ is the control input vector, $V(t) \in R^{n}$ is the weight vector and $F \in R^{m}$ is the fault vector, and $\tau$ is the time-delay term. $\varphi(t)$ is a real valued continuous function. $A \in R^{n \times n}, A_{d} \in R^{n \times n}, B \in R^{n \times m}, D \in$ $R^{n \times n}, B_{d} \in R^{n \times m}, E \in R^{n \times n}$, and $N \in R^{n \times m}$ are system parameter matrices with $\operatorname{rank}(E)=q<n$. Equation (2) represents the static model of the output PDF approximated by the square-root B-spline model. It is denoted that

$$
\begin{aligned}
C(y) & =\left[\phi_{1}(y), \phi_{2}(y), \ldots, \phi_{n}(y)\right], \\
V & =\left[\omega_{1}, \omega_{2}, \ldots, \omega_{n}\right]^{T} \quad(V \neq 0),
\end{aligned}
$$

where $\phi_{i}(y)(i=1,2, \ldots, n, n \geq 2)$ is the prespecified basis function, $\omega_{i}(i=1,2, \ldots, n)$ is the approximation weight which is only related to $u(t)$, and $n$ is the number of basis functions. $d(t)$ is the unknown external disturbance which can be supposed to be generated by a linear exogenous system described by

$$
\begin{aligned}
& \dot{\omega}(t)=W \omega(t), \\
& d(t)=T \omega(t) .
\end{aligned}
$$

Remark 1. From literatures [21], many kinds of disturbances in engineering can be described by this model, for example, unknown constant and harmonics with unknown phase and 
magnitude. In most existing results, the disturbances are restricted to be bounded exogenous signals. The state variable of the exogenous system $\omega(t)$ is the target of estimation, $d(t)$ is the form of disturbance, and $W$ and $T$ are the known parameter matrices with suitable dimensions.

The following assumptions are used throughout this paper.

Assumption 2. $(A, D)$ is observable, and $\left(W, B_{d} T\right)$ is observable.

Assumption 3. The fault and disturbance occurring in SDC system are bounded; that is, $\|F\| \leq M_{f}$ and $\|d(t)\| \leq M_{d}$, where $M_{f}$ and $M_{d}$ are two positive constants.

Assumption 4. The system (1) is regular and impulse-free; that is, $|s E-A| \neq 0$ and $\operatorname{rank}(E)=\operatorname{deg}(|s E-A|)$.

With the assumptions, there exist two matrices $\left(L_{1}, L_{2}\right)$ such that the following equation

$$
\begin{aligned}
L_{1} E L_{2} & =\left[\begin{array}{ll}
I_{q} & 0 \\
0 & 0
\end{array}\right], \\
L_{1} A L_{2} & =\left[\begin{array}{cc}
A_{1} & 0 \\
0 & I_{n-q}
\end{array}\right]
\end{aligned}
$$

holds. It is assumed that $L_{1} A_{d} L_{2}=\left[\begin{array}{cc}A_{d 1} & 0 \\ 0 & A_{d 2}\end{array}\right]$ holds simultaneously. Then the SDC system (1) can be transformed as

$$
\begin{aligned}
\dot{x}_{1}(t)= & A_{1} x_{1}(t)+A_{d 1} x_{1}(t-\tau)+B_{1} u(t)+N_{1} F(t) \\
& +B_{d 1} T \omega(t), \\
x_{2}(t)= & -A_{d 2} x_{2}(t-\tau)-B_{2} u(t)-N_{2} F(t) \\
& -B_{d 2} T \omega(t), \\
V(t)= & D_{1} x_{1}(t)+D_{2} x_{2}(t), \\
\dot{\omega}(t)= & W \omega(t),
\end{aligned}
$$

where $B_{1}, B_{d 1}, N_{1} \in R^{q \times m}, B_{2}, B_{d 2}, N_{2} \in R^{(n-q) \times m}, D_{1} \in$ $R^{(n-1) \times q}$, and $D_{2} \in R^{(n-1) \times(n-q)}$, which can be determined as follows:

$$
\begin{aligned}
L_{2} B & =\left[\begin{array}{l}
B_{1} \\
B_{2}
\end{array}\right], \\
L_{2} B_{d} & =\left[\begin{array}{l}
B_{d 1} \\
B_{d 2}
\end{array}\right], \\
D L_{1} & =\left[\begin{array}{ll}
D_{1} & D_{2}
\end{array}\right], \\
L_{2} N & =\left[\begin{array}{l}
N_{1} \\
N_{2}
\end{array}\right] .
\end{aligned}
$$

\section{Fault Diagnosis}

In order to reject the disturbance and eliminate the influence to the fault diagnosis, a full-order observer is constructed to estimate the external disturbance. Denoting $s(t)=\left[\begin{array}{c}x_{1}(t) \\ \omega(t)\end{array}\right]$, the full-order system is constructed by augmenting state (1) with exogenous system (4) as follows:

$$
\begin{aligned}
\dot{s}(t)= & A_{0} s(t)+A_{d 0} s(t-\tau)+B_{0} u(t)+N_{0} F(t), \\
x_{2}(t)= & -A_{d 2} x_{2}(t-\tau)-B_{2} u(t)-N_{2} F(t) \\
& -B_{d 2} T \omega(t), \\
V(t)= & D_{1} x_{1}(t)+D_{2} x_{2}(t)=D_{0} s(t)+D_{2} x_{2}(t),
\end{aligned}
$$

where

$$
\begin{aligned}
A_{0} & =\left[\begin{array}{cc}
A_{1} & B_{d 1} T \\
0 & W
\end{array}\right], \\
A_{d 0} & =\left[\begin{array}{cc}
A_{d 1} & 0 \\
0 & 0
\end{array}\right], \\
B_{0} & =\left[\begin{array}{c}
B_{1} \\
0
\end{array}\right], \\
N_{0} & =\left[\begin{array}{c}
N_{1} \\
0
\end{array}\right], \\
D_{0} & =\left[\begin{array}{ll}
D_{1} & 0
\end{array}\right] .
\end{aligned}
$$

The full-order observer is designed as follows:

$$
\begin{aligned}
& \dot{\hat{s}}(t)= A_{0} \widehat{s}(t)+A_{d 0} \widehat{s}(t-\tau)+B_{0} u(t) \\
&+K_{1} \varepsilon(t), \\
& \widehat{x}_{2}(t)=-A_{d 2} \widehat{x}_{2}(t-\tau)-B_{2} u(t)-N_{2} F(t) \\
&-B_{d 2} T \widehat{\omega}(t), \\
& \widehat{V}(t)= D_{0} \widehat{s}(t)+D_{2} \widehat{x}_{2}(t), \\
& \sqrt{\widehat{\gamma}(y, u(t))=} \frac{C(y) \widehat{V}(t)}{\sqrt{\widehat{V}^{T}(t) \Sigma_{1} \widehat{V}(t)}}, \\
& \varepsilon(t)=\int_{a}^{b}(\sqrt{\gamma}-\sqrt{\widehat{\gamma}}) d y,
\end{aligned}
$$

where $K_{1}$ is observer gain matrix, $\varepsilon(t)$ is the residual signal, and

$$
\begin{aligned}
\varepsilon(t)= & \frac{\Sigma_{2}\left(D_{0} s(t)+D_{2} x_{2}(t)\right)}{\sqrt{V^{T}(t) \Sigma_{1} V(t)}} \\
& -\frac{\Sigma_{2}\left(D_{0} \widehat{s}(t)+D_{2} \widehat{x}_{2}(t)\right)}{\sqrt{\widehat{V}^{T}(t) \Sigma_{1} \widehat{V}(t)}} \\
& +\frac{\Sigma_{2}\left(D_{0} s(t)+D_{2} x_{2}(t)\right)}{\sqrt{\widehat{V}^{T}(t) \Sigma_{1} \widehat{V}(t)}}
\end{aligned}
$$




$$
\begin{aligned}
& -\frac{\Sigma_{2}\left(D_{0} s(t)+D_{2} x_{2}(t)\right)}{\sqrt{\widehat{V}^{T}(t) \Sigma_{1} \widehat{V}(t)}} \\
= & \frac{\Sigma_{2}\left(D_{0} e_{s}(t)+D_{2} e_{2}(t)\right)}{\sqrt{\widehat{V}^{T}(t) \Sigma_{1} \widehat{V}(t)}} \\
+ & \frac{\Sigma_{2}\left(D_{0} s(t)+D_{2} x_{2}(t)\right)}{\sqrt{\widehat{V}^{T}(t) \Sigma_{1} \widehat{V}(t)}} \\
& \cdot\left(\frac{\sqrt{\widehat{V}^{T}(t) \Sigma_{1} \widehat{V}(t)}-\sqrt{V^{T}(t) \Sigma_{1} V(t)}}{\sqrt{V^{T}(t) \Sigma_{1} V(t)}}\right),
\end{aligned}
$$

where $e_{s}(t)=s(t)-\widehat{s}(t), \Sigma_{2}=\int_{a}^{b} C(y) d y$, and $e_{2}(t)=x_{2}(t)-$ $\hat{x}_{2}(t)$.

Lemma 5 (see [19]). There exists a constant $\lambda$ such that the following equation

$$
\begin{aligned}
& \sqrt{\widehat{\widehat{V}}^{T}(t) \Sigma_{1} \widehat{V}(t)}-\sqrt{V^{T}(t) \Sigma_{1} V(t)} \\
& =\lambda\left(\|\widehat{V}(t)\|-\left\|V^{T}(t)\right\|\right)
\end{aligned}
$$

holds, where $\lambda_{\min }\left(\Sigma_{1}\right) / \lambda_{\max }\left(\Sigma_{1}\right) \leq \lambda \leq \lambda_{\max }\left(\Sigma_{1}\right) / \lambda_{\min }\left(\Sigma_{1}\right)$ and $\lambda_{\max }\left(\Sigma_{1}\right)$ and $\lambda_{\min }\left(\Sigma_{1}\right)$ are the maximum and minimum eigenvalues of matrix $\Sigma_{1}$, respectively.

Then, it can be obtained that

$$
\begin{aligned}
& \varepsilon(t) \\
& =\frac{\Sigma_{2}\left(D_{0} e_{s}(t)+D_{2} e_{2}(t)\right)}{\sqrt{\widehat{V}^{T}(t) \Sigma_{1} \widehat{V}(t)}} \\
& \quad+\frac{\Sigma_{2}\left(D_{0} s(t)+D_{2} x_{2}(t)\right)}{\sqrt{\widehat{\widehat{V}}^{T}(t) \Sigma_{1} \widehat{V}(t)}} \frac{\lambda_{1}\left(\|\widehat{V}(t)\|-\left\|V^{T}(t)\right\|\right)}{\sqrt{V^{T}(t) \Sigma_{1} V(t)}} .
\end{aligned}
$$

The full-order observation error dynamic system can be formulated as follows:

$$
\begin{aligned}
\dot{e}_{s}(t)= & \dot{s}(t)-\dot{\hat{\mathcal{s}}}(t) \\
= & A_{0} e_{s}(t)+A_{d 0} e_{s}(t-\tau)+N_{0} F \\
& -L_{3} \Sigma_{2} D_{0} e_{s}(t)-L_{3} \Sigma_{2} D_{2} e_{2}(t) \\
& +L_{3} \Sigma_{2} V\left(\frac{\lambda_{1}(\|V\|-\|\widehat{V}\|)}{\sqrt{V^{T} \Sigma_{1} V}}\right),
\end{aligned}
$$

where $L_{3}=K_{1} / \sqrt{\widehat{V}^{T}(t) \Sigma_{1} \widehat{V}(t)}$ is the gain matrix to be determined later.
The purpose of fault diagnosis is to estimate the size of fault. The fault diagnosis observer is constructed as follows:

$$
\begin{aligned}
\dot{\hat{x}}_{1}(t)= & A_{1} \widehat{x}_{1}(t)+A_{d 1} \widehat{x}_{1}(t-\tau)+B_{1} u(t) \\
& +N_{1} \widehat{F}(t)+B_{d 1} T \widehat{\omega}(t)+K_{2} \varepsilon(t), \\
\widehat{x}_{2}(t)= & -A_{d 2} \widehat{x}_{2}(t-\tau)-B_{2} u(t)-N_{2} \widehat{F}(t) \\
& -B_{d 2} T \widehat{\omega}(t), \\
\widehat{V}(t)= & D_{1} \widehat{x}_{1}(t)+D_{2} \widehat{x}_{2}(t), \\
\sqrt{\widehat{\gamma}(y, u(t))=} & \frac{C(y) \widehat{V}(t)}{\sqrt{\widehat{V}^{T}(t) \Sigma_{1} \widehat{V}(t)}}, \\
\dot{\hat{F}}= & -\Gamma_{1} \widehat{F}+\Gamma_{2} \varepsilon(t),
\end{aligned}
$$

where $e_{1}(t)=x_{1}(t)-\widehat{x}_{1}(t)$. Then the observation error dynamic system is obtained as follows:

$$
\begin{aligned}
\dot{e}_{1}(t)= & A_{1} e_{1}(t)+A_{d 1} e_{1}(t-\tau)+N_{1} \widetilde{F}(t) \\
& +B_{d 1} T \widetilde{\omega}(t)-L_{4} \Sigma_{2} D_{1} e_{1}-L_{4} \Sigma_{2} D_{2} e_{2} \\
& +L_{4} \Sigma_{2} V\left(\frac{\lambda_{2}(\|V\|-\|\widehat{V}\|)}{\sqrt{V^{T} \Sigma_{1} V}}\right) \\
\dot{\widehat{F}}= & -\Gamma_{1} \widehat{F}+L_{5} \Sigma_{2} D_{1} e_{1}+L_{5} \Sigma_{2} D_{2} e_{2} \\
& -L_{5} \Sigma_{2} V\left(\frac{\lambda_{2}(\|V\|-\|\widehat{V}\|)}{\sqrt{V^{T} \Sigma_{1} V}}\right)
\end{aligned}
$$

where $\widetilde{F}=F-\widehat{F}, \widetilde{\omega}=\omega-\widehat{\omega} \cdot L_{4}=K_{2} / \sqrt{\widehat{V}^{T}(t) \Sigma_{1} \widehat{V}(t)}, L_{5}=$ $\Gamma_{2} / \sqrt{\widehat{V}^{T}(t) \Sigma_{1} \widehat{V}(t)}$, and $\Gamma_{1}$ are gain matrices to be determined later.

Combing (14), (16), and (17), the following augmentation error dynamic system can be obtained as

$$
\begin{aligned}
\left(\begin{array}{c}
\dot{e}_{s}(t) \\
\dot{e}_{1}(t) \\
\dot{\hat{F}}(t)
\end{array}\right)= & \bar{A}\left(\begin{array}{c}
e_{s}(t) \\
e_{1}(t) \\
\widehat{F}(t)
\end{array}\right)+\left(\begin{array}{c}
A_{d 0} e_{s}(t-\tau) \\
A_{d 1} e_{1}(t-\tau) \\
0
\end{array}\right) \\
& +\left(\begin{array}{c}
N_{0} F(t) \\
N_{1} F(t) \\
0
\end{array}\right)+\left(\begin{array}{c}
-L_{3} \Sigma_{2} D_{2} e_{2} \\
-L_{4} \Sigma_{2} D_{2} e_{2} \\
L_{5} \Sigma_{2} D_{2} e_{2}
\end{array}\right) \\
& +\left(\begin{array}{c}
L_{3} \Sigma_{2} h_{1} \\
L_{4} \Sigma_{2} h_{2} \\
-L_{5} \Sigma_{2} h_{2}
\end{array}\right),
\end{aligned}
$$


where

$$
\begin{aligned}
& \bar{A}=\left[\begin{array}{ccc}
A_{0}-L_{3} \Sigma_{2} D_{0} & 0 & 0 \\
\bar{T} & A_{1}-L_{4} \Sigma_{2} D_{1} & -N_{1} \\
0 & L_{5} & -\Gamma_{1}
\end{array}\right], \\
& \bar{T}=\left[\begin{array}{ll}
0 & B_{d 1} T
\end{array}\right], \\
& h_{i}=V\left(\frac{\lambda_{i}(\|V\|-\|\widehat{V}\|)}{\sqrt{V^{T} \Sigma_{1} V}}\right), \quad i=1,2 .
\end{aligned}
$$

Select the reference output as follows:

$$
\begin{aligned}
s_{\infty}= & C_{1} e_{s}(t)+C_{2} e_{1}(t)+C_{3} e_{1}(t-\tau)+C_{4} \widetilde{F} \\
& +C_{5} e_{2}(t) .
\end{aligned}
$$

For the constants $\mu_{1}>0$ and $\mu_{2}>0$, the generalized $H_{\infty}$ performance is denoted as follows:

$$
\begin{aligned}
J_{\infty}= & \left\|s_{\infty}\right\|^{2}-\mu_{1}^{2}\|F(t)\|^{2}-\mu_{2}^{2}\left\|e_{2}(t)\right\|^{2} \\
& -\delta\left(Q_{1}, Q_{2}, Q_{3}, Q_{4}\right), \\
\delta\left(Q_{1}, Q_{2}, Q_{3}, Q_{4}\right)= & e_{s}^{T}(0) Q_{1} e_{s}(0)+e_{1}^{T}(0) Q_{2} e_{1}(0) \\
& +\int_{-\tau}^{0} \varphi_{1}^{T} Q_{3} \varphi_{1} d \alpha \\
& +\int_{-\tau}^{0} \varphi_{2}^{T} Q_{4} \varphi_{2} d \alpha .
\end{aligned}
$$

Theorem 6. For the matrices $C_{i}>0(i=1,2,3,4,5)$ and $Q_{i}>0(i=1,2,3,4)$ and constants $\mu_{i}>0(i=1,2)$, there exist positive definite symmetric matrices $P_{1}$ and $P_{2}$, positive definite matrices $R_{1}$ and $R_{2}$, and gain matrices $L_{3}, L_{4}, L_{5}$, and $\Gamma_{1}$ satisfying $P_{1} \leq Q_{1}, P_{2} \leq Q_{2}, R_{1} \leq Q_{3}, R_{2} \leq Q_{4}$, and the following LMI, and then the augmenting error dynamic system (18) is stable and satisfies $J_{\infty}<0$

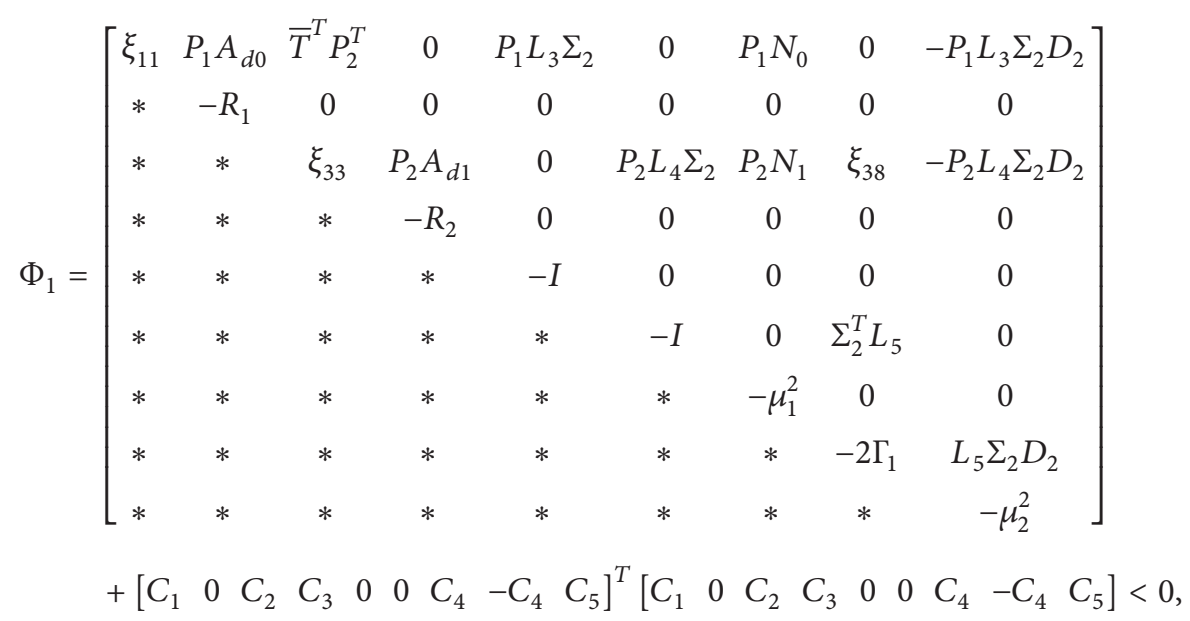

where

$$
\begin{aligned}
\xi_{11}= & \left(A_{0}-L_{3} \Sigma_{2} D_{0}\right)^{T} P_{1}+P_{1}\left(A_{0}-L_{3} \Sigma_{2} D_{0}\right)+R_{1} \\
& +m_{1} I, \\
\xi_{33}= & \left(A_{1}-L_{4} \Sigma_{2} D_{1}\right)^{T} P_{2}+P_{2}\left(A_{1}-L_{4} \Sigma_{2} D_{1}\right)+R_{2} \\
& +m_{2} I, \\
\xi_{38}= & D_{1}^{T} \Sigma_{2}^{T} L_{5}^{T}-P_{2} N_{1} .
\end{aligned}
$$

Proof. Select the following Lyapunov functions $\pi_{1}, \pi_{2}$, and $\pi_{3}$ as follows:

$$
\begin{aligned}
\pi_{1}= & e_{s}^{T} P_{1} e_{s}+\int_{0}^{t}\left[m_{1} e_{s}^{T}(\alpha) e_{s}(\alpha)-h_{1}^{T} h_{1}\right] d \alpha \\
& +\int_{t-\tau}^{t} e_{s}^{T}(\alpha) R_{1} e_{s}(\alpha) d \alpha,
\end{aligned}
$$

$$
\begin{aligned}
\pi_{2}= & e_{1}^{T} P_{2} e_{1}+\int_{0}^{t}\left[m_{2} e_{1}^{T}(\alpha) e_{1}(\alpha)-h_{2}^{T} h_{2}\right] d \alpha \\
& +\int_{t-\tau}^{t} e_{1}^{T}(\alpha) R_{2} e_{1}(\alpha) d \alpha, \\
\pi_{3}= & \widehat{F}^{T} \widehat{F} .
\end{aligned}
$$

It can be obtained that the first-order derivatives of $\pi_{1}, \pi_{2}$, and $\pi_{3}$ are given as follows:

$$
\begin{aligned}
\dot{\pi}_{1} & =e_{s}^{T}\left[\left(A_{0}-L_{3} \Sigma_{2} D_{0}\right)^{T} P_{1}+P_{1}\left(A_{0}-L_{3} \Sigma_{2} D_{0}\right)\right. \\
& \left.+R_{1}+m_{1} I\right] e_{s}+2 e_{s}^{T} P_{1} A_{d 0} e_{s}(t-\tau)+2 e_{s}^{T} P_{1} N_{0} F \\
& -2 e_{s}^{T} P_{1} L_{3} \Sigma_{2} D_{2} e_{2}+2 e_{s}^{T} P_{1} L_{3} \Sigma_{2} h_{1}-h_{1}^{T} h_{1}-e_{s}^{T}(t \\
& -\tau) R_{1} e_{s}(t-\tau),
\end{aligned}
$$




$$
\begin{aligned}
\dot{\pi}_{2} & =e_{1}^{T}\left[\left(A_{1}-L_{4} \Sigma_{2} D_{1}\right)^{T} P_{2}+P_{2}\left(A_{1}-L_{4} \Sigma_{2} D_{1}\right)\right. \\
& \left.+R_{2}+m_{2} I\right] e_{1}+2 e_{1}^{T} P_{2} \bar{T} e_{s}+2 e_{1}^{T} P_{2} N_{1} F \\
& +2 e_{1}^{T} P_{2} A_{d 1} e_{1}(t-\tau)-2 e_{1}^{T} P_{2} L_{4} \Sigma_{2} D_{2} e_{2} \\
& -2 e_{1}^{T} P_{2} N_{1} \widehat{F}+2 e_{1}^{T} P_{2} L_{4} \Sigma_{2} h_{2}-h_{2}^{T} h_{2}-e_{1}^{T}(t-\tau) \\
& \cdot R_{2} e_{1}(t-\tau), \\
\dot{\pi}_{3} & =-2 \Gamma_{1} \widehat{F}^{T} \widehat{F}+2 e_{1}^{T} D_{1}^{T} \Sigma_{2}^{T} L_{5}^{T} \widehat{F}+2 \widehat{F}^{T} L_{5} \Sigma_{2} D_{2} e_{2} \\
& +2 \widehat{F}^{T} L_{5} \Sigma_{2} h_{2} .
\end{aligned}
$$

Denote $\pi=\pi_{1}+\pi_{2}+\pi_{3}$, and consider an auxiliary function as the performance index

$$
\begin{aligned}
J_{\mathrm{aux}} & =\int_{0}^{t}\left(\left\|s_{\infty}\right\|^{2}-\mu_{1}^{2}\|F(\alpha)\|^{2}-\mu_{2}^{2}\left\|e_{2}(\alpha)\right\|^{2}\right. \\
& +\dot{\pi}(\alpha)) d \alpha .
\end{aligned}
$$

Then it can be obtained that $\left\|s_{\infty}\right\|^{2}-\mu_{1}^{2}\|F(t)\|^{2}-\mu_{2}^{2}\left\|e_{2}(t)\right\|^{2}+$ $\dot{\pi}=q^{T} \Phi_{1} q$, where

$$
q^{T}=\left[\begin{array}{llllllllll}
e_{s}^{T}(t) & e_{s}^{T}(t-\tau) & e_{1}^{T}(t) & e_{1}^{T}(t-\tau) & h_{1}^{T}(t) & h_{2}^{T}(t) & F^{T}(t) & \widehat{F}^{T}(t) & e_{2}^{T}(t)
\end{array}\right]^{T} .
$$

From the Schur complement lemma, $\Phi_{1}$ equals inequality (22), leading to $J_{\text {aux }}<0$. Then

$$
\begin{aligned}
J_{\mathrm{aux}} & =\int_{0}^{t}\left(\left\|s_{\infty}\right\|^{2}-\mu_{1}^{2}\|F(t)\|^{2}-\mu_{2}^{2}\left\|e_{2}(t)\right\|^{2}\right) d \tau \\
& +\pi(t)-\pi(0) \leq\left\|s_{\infty}\right\|^{2}-\mu_{1}^{2}\|F(t)\|^{2}-\mu_{2}^{2}\left\|e_{2}(t)\right\|^{2} \\
& -\left[e_{s}^{T}(0) P_{1} e_{s}(0)+e_{1}^{T}(0) P_{2} e_{1}(0)\right. \\
& \left.+\int_{-\tau}^{0} \varphi_{1}^{T} R_{1} \varphi_{1} d \alpha+\int_{-\tau}^{0} \varphi_{2}^{T} R_{2} \varphi_{2} d \alpha\right]
\end{aligned}
$$

where when $t \in[-\tau, 0], \widehat{s}=0, \widehat{x}_{1}=0, s=\varphi_{1}(t)$, and $x_{1}=$ $\varphi_{2}(t)$. It can be obtained that

$$
\begin{aligned}
& J_{\infty}<J_{\text {aux }}+\delta\left(P_{1}-Q_{1}, P_{2}-Q_{2}, R_{1}-Q_{3}, R_{2}-Q_{4}\right), \\
& \delta\left(P_{1}-Q_{1}, P_{2}-Q_{2}, R_{1}-Q_{3}, R_{2}-Q_{4}\right) \\
& \quad=\left[e_{s}^{T}(0) P_{1} e_{s}(0)+e_{1}^{T}(0) P_{2} e_{1}(0)\right. \\
& \left.\quad+\int_{-\tau}^{0} \phi_{1}^{T} R_{1} \phi_{1} d \alpha+\int_{-\tau}^{0} \phi_{2}^{T} R_{2} \phi_{2} d \alpha\right] \\
& \quad-\left[e_{s}^{T}(0) Q_{1} e_{s}(0)+e_{1}^{T}(0) Q_{2} e_{1}(0)\right. \\
& \left.\quad+\int_{-\tau}^{0} \phi_{1}^{T} Q_{3} \phi_{1} d \alpha+\int_{-\tau}^{0} \phi_{2}^{T} Q_{4} \phi_{2} d \alpha\right] .
\end{aligned}
$$

Since $\delta\left(P_{1}-Q_{1}, P_{2}-Q_{2}, R_{1}-Q_{3}, R_{2}-Q_{4}\right)<0, J_{\infty}<0$ is proved. This completes the proof.

\section{Fault Tolerant Control}

After estimating the fault and disturbance, it is necessary to design a fault tolerant controller to make the postfault output
PDF still track the desired PDF. A given desired PDF can be described as follows:

$$
\sqrt{\gamma_{g}(y)}=\frac{C(y) V_{g}}{\sqrt{V_{g}^{T} \Sigma_{1} V_{g}}}, \quad \forall y \in[a, b],
$$

where $V_{g}$ is the expected weight vector of desired PDF $\gamma_{g}(y)$. Let

$$
\begin{aligned}
e_{2}(t) & =V(t)-V_{g}, \\
E D^{-1} \dot{e}_{2}(t) & =E D^{-1}\left(\dot{V}(t)-\dot{V}_{g}\right)=E \dot{e}_{m}(t) \\
& =E\left(\dot{x}(t)-\dot{x}_{g}\right) .
\end{aligned}
$$

Then the tracking error dynamic system can be expressed as follows:

$$
\begin{aligned}
E \dot{e}_{m}(t)= & A e_{m}(t)+A_{d} e_{m}(t-\tau)+B u(t)+N F(t) \\
& +B_{d} d(t)+\left(A+A_{d}\right) x_{g} .
\end{aligned}
$$

Denote

$$
\begin{array}{r}
\bar{E}=L_{1} E L_{2}=\left[\begin{array}{ll}
I_{q} & 0 \\
0 & 0
\end{array}\right], \\
L_{2}^{-1} e_{m}(t)=\left[\begin{array}{l}
\xi_{1}(t) \\
\xi_{2}(t)
\end{array}\right]=\xi(t) .
\end{array}
$$

Then an augmentation control input containing the system input and the information of desired PDF is constructed as $U(t)=u(t)+\left(B^{T} B\right)^{-1} B^{T}\left(A+A_{d}\right) x_{g}$, where the former item is the reconstructed control input and the latter is the extended information of desired PDF. The tracking error dynamic system can be rewritten as follows:

$$
\begin{aligned}
\bar{E} \dot{\xi}(t)= & L_{1} A L_{2} \xi(t)+L_{1} A_{d} L_{2} \xi(t-\tau)+L_{1} B U(t) \\
& +L_{1} N F(t)+L_{1} B_{d} d(t) .
\end{aligned}
$$


Assume that $U(t)$ is the feedback of the tracking error, fault, and disturbance, then it can be obtained that

$$
\begin{aligned}
U(t)= & \Gamma_{3} \int_{a}^{b}(\sqrt{\gamma(y, u(t))}-\sqrt{g(y)}) d y+\Gamma_{4} F(t) \\
& +\Gamma_{5} d(t) \\
= & \frac{\Gamma_{3} \Sigma_{2} D L_{2} \xi(t)}{\sqrt{V_{g}^{T} \Sigma_{1} V_{g}}+\frac{\Gamma_{3} \Sigma_{2} V(t)}{\sqrt{V_{g}^{T} \Sigma_{1} V_{g}}}} \\
& \cdot \frac{\lambda_{3}\left(\left\|V_{g}\right\|-\|V(t)\|\right)}{\sqrt{V^{T}(t) \Sigma_{1} V(t)}+\Gamma_{4} F(t)+\Gamma_{5} d(t)} \\
= & L_{6} \Sigma_{2} D L_{2} \xi(t)+L_{6} \Sigma_{2} h_{3}+\Gamma_{4} F(t)+\Gamma_{5} d(t),
\end{aligned}
$$

where $L_{6}=\Gamma_{3} / \sqrt{V_{g}^{T} \Sigma_{1} V_{g}}$ and $h_{3}=\left(\lambda_{3}\left(\left\|V_{g}\right\|-\right.\right.$ $\left.\|V(t)\|) / \sqrt{V^{T}(t) \Sigma_{1} V(t)}\right) V(t)$.

Theorem 7. For the given constant $\eta_{i}(i=1, \ldots, 4)$ and a small positive constant $\lambda$, suppose that there exist positive definite symmetric matrices $P$ and matrices $\Gamma_{3}, \Gamma_{4}$, and $\Gamma_{5}$ such that the following LMI holds, and then the tracking error dynamic system (34) is stable.

$$
\Phi_{4}=\left[\begin{array}{ccccc}
M_{4} & A_{d} P^{T} & B L_{6} \Sigma_{2} L_{2}^{-1} P^{T} & \frac{1}{\eta_{2}} B \Gamma_{4} & \frac{1}{\eta_{4}} B \Gamma_{5} \\
* & -R_{1} & 0 & 0 & 0 \\
* & * & -I & 0 & 0 \\
* & * & * & -I & 0 \\
* & * & * & * & -I
\end{array}\right]
$$$$
<0 \text {, }
$$

$$
\begin{aligned}
M_{4}= & A P^{T}+P A^{T}+P L_{2}^{-T} R_{1} L_{2}^{-1} P^{T}+2 B L_{6} \Sigma_{2} D P^{T} \\
& +P L_{2}^{-T} \alpha_{3} L_{2}^{-1} P^{T}+\frac{1}{\eta_{1}^{2}} N N^{T}+\frac{1}{\eta_{3}^{2}} B_{d} B_{d}^{T}+\lambda I, \\
\Gamma_{3}= & L_{6} \sqrt{V_{g}^{T} \Sigma_{1} V_{g} .}
\end{aligned}
$$

Proof. Select the following Lyapunov function as follows:

$$
\begin{aligned}
\pi_{4}= & \xi^{T} \bar{E}^{T} \bar{P}^{-T} \xi+\int_{t-\tau}^{t} \xi^{T}(s) R_{1} \xi(s) d s \\
& +\int_{0}^{t}\left(\alpha_{3} \xi^{T} \xi-h_{3}^{T} h_{3}\right) d s,
\end{aligned}
$$

where $h_{3}=\left(\lambda_{3}\left(\left\|V_{g}\right\|-\|V(t)\|\right) / \sqrt{V^{T}(t) \Sigma_{1} V(t)}\right) V(t)$. It can be obtained that $h_{3}^{T} h_{3} \leq\left(\lambda_{3}\left\|D L_{2}\right\| / \sqrt{\left\|\Sigma_{1}\right\|}\right)^{2} \xi^{T}(t) \xi(t)$, and denote $\alpha_{3}=\left(\lambda_{3}\left\|D L_{2}\right\| / \sqrt{\left\|\Sigma_{1}\right\|}\right)^{2}$; then $\pi_{4} \geq 0$ holds. The firstorder derivative of $\pi_{4}$ can be obtained as follows:

$$
\begin{aligned}
& \dot{\pi}_{4}=\xi^{T}\left[\bar{P}^{-1} L_{1} A L_{2}+L_{2}^{T} A^{T} L_{1}^{T} \bar{P}^{-T}+R_{1}\right. \\
& \left.+2 \bar{P}^{-1} L_{1} B L_{6} \Sigma_{2} D L_{2}\right] \xi+\alpha_{3} \xi^{T} \xi \\
& +2 \xi^{T} \bar{P}^{-1} L_{1} A_{d} L_{2} \xi(t-\tau)-h_{3}^{T} h_{3}+2 \xi^{T}\left(\bar{P}^{-1} L_{1} N\right. \\
& \left.+\bar{P}^{-1} L_{1} B \Gamma_{4}\right) F+2 \xi^{T} \bar{P}^{-1} L_{1} B_{d} d(t) \\
& +2 \xi^{T} \bar{P}^{-1} L_{1} B \Gamma_{5} d(t)-\xi^{T}(t-\tau) R_{1} \xi(t-\tau) \\
& +2 \xi^{T} \bar{P}^{-1} L_{1} B L_{6} \Sigma_{2} h_{3} \leq \xi^{T}\left[\bar{P}^{-1} L_{1} A L_{2}\right. \\
& \left.+L_{2}^{T} A^{T} L_{1}^{T} \bar{P}^{-T}+R_{1}+2 \bar{P}^{-1} L_{1} B L_{6} \Sigma_{2} D L_{2}+\alpha_{3}\right] \xi \\
& +\eta_{4}^{2} d^{T}(t) d(t)+2 \xi^{T} \bar{P}^{-1} L_{1} A_{d} L_{2} \xi(t-\tau) \\
& -\xi^{T}(t-\tau) R_{1} \xi(t-\tau)+2 \xi^{T} \bar{P}^{-1} L_{1} B L_{6} \Sigma_{2} h_{3} \\
& -h_{3}^{T} h_{3}+\frac{1}{\eta_{1}^{2}} \xi^{T} \bar{P}^{-1} L_{1} N\left(\bar{P}^{-1} L_{1} N\right)^{T} \xi+\eta_{1}^{2} F^{T} F \\
& +\frac{1}{\eta_{2}^{2}} \xi^{T} \bar{P}^{-1} L_{1} B \Gamma_{4}\left(\bar{P}^{-1} L_{1} B \Gamma_{4}\right)^{T} \xi+\eta_{2}^{2} F^{T} F+\frac{1}{\eta_{3}^{2}} \\
& \cdot \xi^{T} \bar{P}^{-1} L_{1} B_{d}\left(\bar{P}^{-1} L_{1} B_{d}\right)^{T} \xi+\eta_{3}^{2} d^{T}(t) d(t)+\frac{1}{\eta_{4}^{2}} \\
& \cdot \xi^{T} \bar{P}^{-1} L_{1} B \Gamma_{5}\left(\bar{P}^{-1} L_{1} B \Gamma_{5}\right)^{T} \xi=q^{T} \Phi_{1} q+\left(\eta_{1}^{2}\right. \\
& \left.+\eta_{2}^{2}\right) F^{T} F+\left(\eta_{3}^{2}+\eta_{4}^{2}\right) d^{T}(t) d(t)
\end{aligned}
$$

where $q^{T}=\left[\begin{array}{lll}\xi^{T}(t) & \xi^{T}(t-\tau) & h_{3}^{T}\end{array}\right]$ and

$$
\begin{aligned}
\Phi_{1}= & {\left[\begin{array}{ccc}
M_{1} & \bar{P}^{-1} L_{1} A_{d} L_{2} & \bar{P}^{-1} L_{1} B L_{6} \Sigma_{2} \\
* & -R_{1} & 0 \\
* & * & -I
\end{array}\right], } \\
M_{1}= & \bar{P}^{-1} L_{1} A L_{2}+L_{2}^{T} A^{T} L_{1}^{T} \bar{P}^{-T}+R_{1}+\alpha_{3} \\
& +2 \bar{P}^{-1} L_{1} B L_{6} \Sigma_{2} D L_{2} \\
& +\frac{1}{\eta_{1}^{2}} \bar{P}^{-1} L_{1} N\left(\bar{P}^{-1} L_{1} N\right)^{T} \\
& +\frac{1}{\eta_{2}^{2}} \bar{P}^{-1} L_{1} B \Gamma_{4}\left(\bar{P}^{-1} L_{1} B \Gamma_{4}\right)^{T} \\
& +\frac{1}{\eta_{3}^{2}} \bar{P}^{-1} L_{1} B_{d}\left(\bar{P}^{-1} L_{1} B_{d}\right)^{T} \\
& +\frac{1}{\eta_{4}^{2}} \bar{P}^{-1} L_{1} B \Gamma_{5}\left(\bar{P}^{-1} L_{1} B \Gamma_{5}\right)^{T} .
\end{aligned}
$$


Substituting $\bar{P}=L_{1} P L_{2}^{-T}$ and $\bar{P}^{-1}=L_{2}^{T} P^{-1} L_{1}^{-1}$ into $\Phi_{1}$ and premultiplying $\operatorname{diag}\left(P L_{2}^{-T} \quad I \quad I\right)$ and postmultiplying $\operatorname{diag}\left(L_{2}^{-1} P^{T} I \quad I\right)$ to the left and the right side of $\Phi_{1}$, it can be obtained that

$$
\begin{aligned}
\Phi_{2}= & {\left[\begin{array}{ccc}
M_{2} & A_{d} P^{T} & B L_{6} \Sigma_{2} L_{2}^{-1} P^{T} \\
* & -R_{1} & 0 \\
* & * & -I
\end{array}\right], } \\
M_{2}= & A P^{T}+P A^{T}+P L_{2}^{-T} R_{1} L_{2}^{-1} P^{T}+2 B L_{6} \Sigma_{2} D P^{T} \\
& +P L_{2}^{-T} \alpha_{3} L_{2}^{-1} P^{T}+\frac{1}{\eta_{1}^{2}} N N^{T}+\frac{1}{\eta_{2}^{2}} B \Gamma_{4} \Gamma_{4}^{T} B^{T} \\
& +\frac{1}{\eta_{3}^{2}} B_{d} B_{d}^{T}+\frac{1}{\eta_{4}^{2}} B \Gamma_{5} \Gamma_{5}^{T} B^{T} .
\end{aligned}
$$

Adding $\lambda I$ to $M_{2}$, then

$$
\begin{aligned}
\Phi_{3} & =\left[\begin{array}{ccc}
M_{3} & A_{d} P^{T} & B L_{6} \Sigma_{2} L_{2}^{-1} P^{T} \\
* & -R_{1} & 0 \\
* & * & -I
\end{array}\right], \\
M_{3} & =M_{2}+\lambda I .
\end{aligned}
$$

When $\Phi_{3}<0$, it can be acquired that $\dot{\pi}_{4} \leq-\lambda \xi^{T} \xi+$ $\left(\eta_{1}^{2}+\eta_{2}^{2}\right) F^{T} F+\left(\eta_{3}^{2}+\eta_{4}^{2}\right) d^{T}(t) d(t)$. According to the Schur complement lemma, $\Phi_{3}<0$ is equivalent to $\Phi_{4}<0$. Thus, when $\Phi_{4}<0$ holds,

$$
\|\xi\|^{2}>\frac{M_{f}^{2}\left(\eta_{1}^{2}+\eta_{2}^{2}\right)+M_{d}^{2}\left(\eta_{3}^{2}+\eta_{4}^{2}\right)}{\lambda}
$$

is satisfied, and then $\dot{\pi}_{4}<0$, leading to

$$
\|\xi\|^{2} \leq \frac{M_{f}^{2}\left(\eta_{1}^{2}+\eta_{2}^{2}\right)+M_{d}^{2}\left(\eta_{3}^{2}+\eta_{4}^{2}\right)}{\lambda},
$$

and the tracking error dynamic system (34) is stable.

By substituting $\widehat{F}(t)$ and $\widehat{d}(t)$ into (35), the practical fault tolerant tracking controller can be formulated as follows:

$$
\begin{aligned}
u(t)= & U(t)-\left(B^{T} B\right)^{-1} B^{T}\left(A+A_{d}\right) \widehat{x}_{g} \\
= & L_{6} \Sigma_{2} D L_{2} \xi(t)+L_{6} \Sigma_{2} h_{3}+\Gamma_{4} \widehat{F}(t)+\Gamma_{5} \widehat{d}(t) \\
& -\left(B^{T} B\right)^{-1} B^{T}\left(A+A_{d}\right) \widehat{x}_{g} .
\end{aligned}
$$

\section{A Simulation Example}

To illustrate the effectiveness of the proposed algorithms, a SDC system whose output PDF can be approximated by the following B-spline functions $\phi_{i}(y), i=1,2,3$, is considered as

$$
\begin{aligned}
\phi_{1}(y)= & 0.5(y-2)^{2} I_{1}+\left(-y^{2}+7 y-11.5\right) I_{2} \\
& +0.5(y-5)^{2} I_{3}, \\
\phi_{2}(y)= & 0.5(y-3)^{2} I_{2}+\left(-y^{2}+9 y-19.5\right) I_{3} \\
& +0.5(y-6)^{2} I_{4}, \\
\phi_{3}(y)= & 0.5(y-4)^{2} I_{3}+\left(-y^{2}+11 y-29.5\right) I_{4} \\
& +0.5(y-7)^{2} I_{5},
\end{aligned}
$$

where $I_{i}(i=1,2, \ldots, 5)$ is an interval function defined as

$$
I_{i}=\left\{\begin{array}{ll}
1, & y \in[i+1, i+2] \\
0, & \text { otherwise },
\end{array} \quad i=1,2, \ldots, 5\right.
$$

The system parameter matrices of the SDC system (1) are given as follows:

$$
\begin{aligned}
& E=\left[\begin{array}{lll}
1 & 0 & 0 \\
0 & 1 & 0 \\
0 & 0 & 0
\end{array}\right], \\
& A=\left[\begin{array}{ccc}
-3.2 & 0.9 & -0.2 \\
0.3 & -3.51 & -0.1 \\
0 & 0 & -1
\end{array}\right],
\end{aligned}
$$$$
A_{d}=\left[\begin{array}{ccc}
-0.1 & -0.5 & 0.12 \\
0.2 & -0.25 & 0.06 \\
0 & 0 & 0.6
\end{array}\right] \text {, }
$$

$$
H=\left[\begin{array}{l}
0.1 \\
0.3 \\
0.3
\end{array}\right] \text {, }
$$

$$
B=\left[\begin{array}{ccc}
1 & 1 & -0.01 \\
0 & 2 & -0.02 \\
0.002 & 0.005 & 0.1
\end{array}\right] \text {, }
$$$$
D=\left[\begin{array}{lll}
1 & 0 & 0 \\
0 & 1 & 0 \\
0 & 0 & 1
\end{array}\right] \text {, }
$$

$$
B_{d}=\left[\begin{array}{ccc}
0.1 & 0.1 & -0.01 \\
0 & 0.2 & -0.02 \\
0.002 & 0.005 & 0.1
\end{array}\right] \text {, }
$$




$$
\begin{aligned}
& L_{1}=\left[\begin{array}{ccc}
1.0 & 0 & -0.2 \\
0 & 1.0 & -0.1 \\
0 & 0 & 1.0
\end{array}\right], \\
& L_{2}=\left[\begin{array}{lll}
1 & 0 & 0 \\
0 & 1 & 0 \\
0 & 0 & 1
\end{array}\right] .
\end{aligned}
$$

The exogenous disturbance can be described by (4), and the corresponding parameter matrices are given as follows:

$$
\begin{gathered}
T=\left[\begin{array}{lll}
2 & 0 & 0 \\
0 & 0 & 0 \\
0 & 0 & 0
\end{array}\right], \\
W=\left[\begin{array}{ccc}
0 & 4 & 0 \\
-4 & 1 & 0 \\
0 & 0 & 2
\end{array}\right] .
\end{gathered}
$$

The time-delay $\tau$ is selected as $\tau=2 \mathrm{~s}$ and the sampling time is chosen as $0.1 \mathrm{~s}$. It is assumed that the fault is constructed as follows:

$$
F= \begin{cases}0, & t<5 \mathrm{~s} \\ 0.5, & t>5 \mathrm{~s} .\end{cases}
$$

The reference output is denoted such that

$$
\begin{aligned}
& C_{1}=\left[\begin{array}{lllll}
0.1 & 0.1 & 0.1 & 0.1 & 0.1
\end{array}\right] \\
& C_{2}=C_{3}=\left[\begin{array}{ll}
0.2 & 0.2
\end{array}\right] \text {, } \\
& C_{4}=C_{5}=0.1 .
\end{aligned}
$$

It can be calculated from the LMIs of Theorems 6 and 7 that

$$
\begin{aligned}
& P_{1} \\
& =\left[\begin{array}{ccccc}
1.8716 & 0.1950 & -0.5180 & 0.5660 & 0.002 \\
0.1950 & 2.0251 & -0.0773 & 0.1075 & 0.003 \\
-0.5180 & -0.0773 & 6.8373 & -0.0841 & -0.0026 \\
0.5660 & 0.1075 & -0.0841 & 6.9108 & 0.0017 \\
0.0002 & 0.0003 & -0.0026 & 0.0017 & 0.0956
\end{array}\right], \\
& P_{2}=\left[\begin{array}{ccccc}
1.160 & 0.0599 \\
0.0599 & 2.1566
\end{array}\right], \\
& \left.R_{1} \quad \begin{array}{ccccc}
7.0146 & -0.0797 & -1.4354 & 1.8075 & -0.0037 \\
-0.0797 & 7.5148 & 0.0394 & 0.1264 & -0.0035 \\
-1.4354 & 0.0394 & 1.3237 & -0.3724 & 0.0004 \\
1.8075 & 0.1264 & -0.3724 & 1.4959 & -0.0011 \\
-0.0037 & -0.0035 & 0.0004 & -0.0011 & 1.1477
\end{array}\right],
\end{aligned}
$$

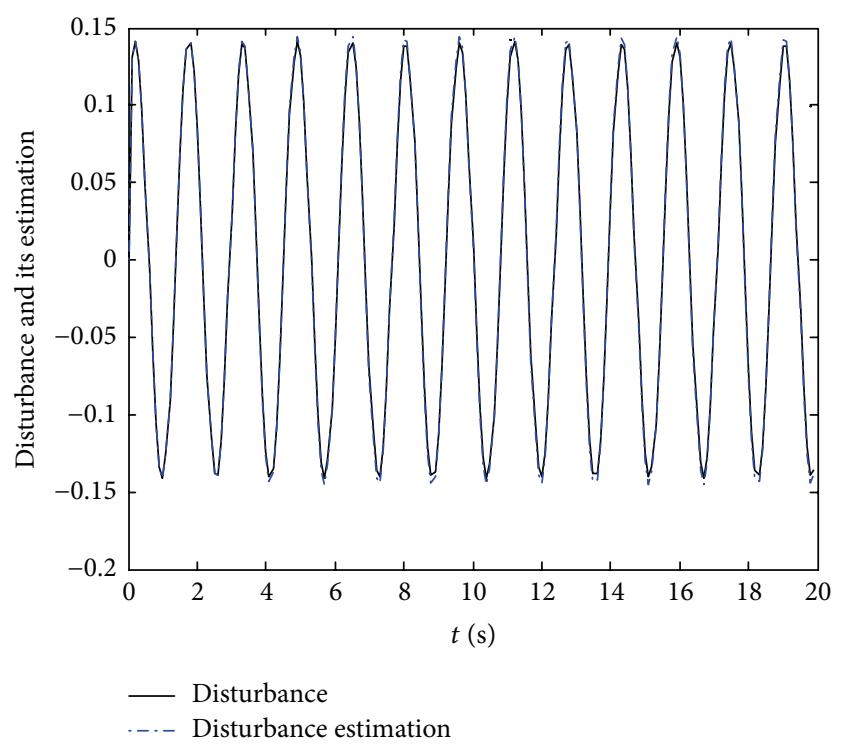

FIGURE 1: Disturbance and its estimation.

$$
\begin{aligned}
& R_{2}=\left[\begin{array}{cc}
2.6380 & -0.5060 \\
-0.5060 & 7.5992
\end{array}\right], \\
& L_{3}=\left[\begin{array}{lllll}
0.0090 & -0.0316 & 0.0109 & -0.0143 & 0
\end{array}\right]^{T} \text {, } \\
& L_{4}=\left[\begin{array}{ll}
0.2435 & -0.0223
\end{array}\right]^{T} \text {, } \\
& L_{5}=0.1038 \text {, } \\
& \Gamma_{1}=0.01 \text {, } \\
& \Gamma_{2}=-0.7 \text {, }
\end{aligned}
$$$$
P=\left[\begin{array}{ccc}
0.1201 & 0.0134 & 0 \\
0.0134 & 0.1206 & 0 \\
0 & 0 & 0.3652
\end{array}\right],
$$$$
\Gamma_{3}=\left[\begin{array}{lll}
-0.0169 & -0.2712 & -3.1352
\end{array}\right] \text {, }
$$$$
\Gamma_{4}=\left[\begin{array}{lll}
0.5301 & -0.1530 & 0.1035
\end{array}\right] \text {, }
$$$$
\Gamma_{5}=\left[\begin{array}{ccc}
0.2905 & -0.0639 & 0.0098 \\
-0.0639 & 0.0669 & 0.0206 \\
0.0098 & 0.0206 & 0.8149
\end{array}\right] \text {. }
$$

The disturbance and its estimation have been shown in Figure 1. The fault diagnosis result has been presented in Figure 2. It can be seen that the desired fault diagnosis result has been obtained and the influence of disturbance has been rejected. The initial PDF, the desired PDF, and the final PDF with fault tolerant control are given in Figure 3. In Figures 4 and 5, the three-dimensional mesh plot shows the changes of the output PDF without and with fault tolerant control, respectively. Before fault occurs, the fault tolerant controller has not been reconstructed, so the disturbance cannot be 


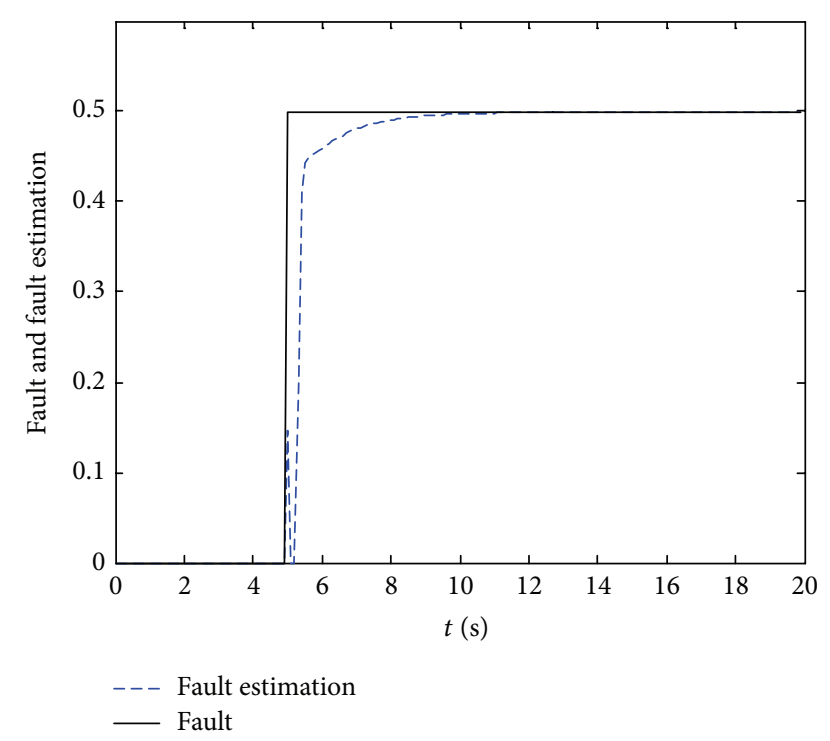

FIgURE 2: Fault and its estimation.

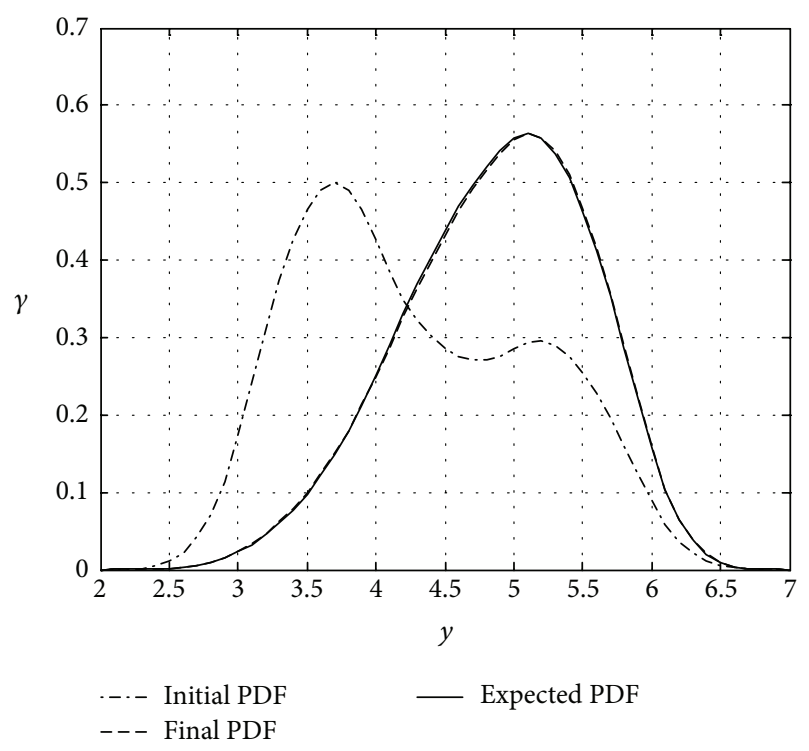

FIgUre 3: The output PDF with fault tolerant control when fault occurs.

rejected. The influence of disturbance is shown in Figure 4. By comparing Figures 4 and 5, the effectiveness of the fault tolerant control can be seen.

\section{Conclusions}

In this paper, a fault diagnosis and fault tolerant control algorithm is given for the non-Gaussian singular timedelayed SDC system based on the rational square-root Bspline approximation model. The external disturbance is taken into consideration. A full-order observer is designed to estimate the disturbance, and then an adaptive observer is constructed to estimate the fault. Using the feedback of output PDF tracking error and the estimation of fault and disturbance, the augmentation control input is designed to

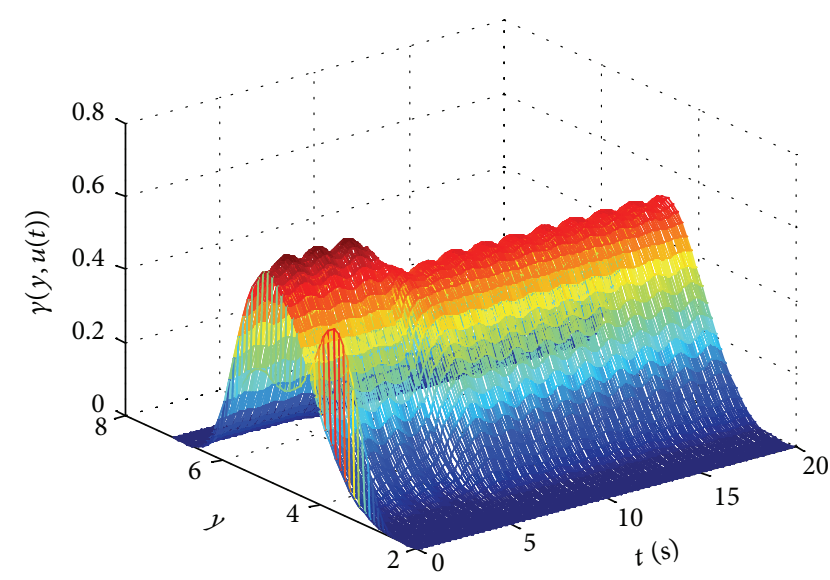

FIgURE 4: The output PDF of the whole process without fault tolerant control.

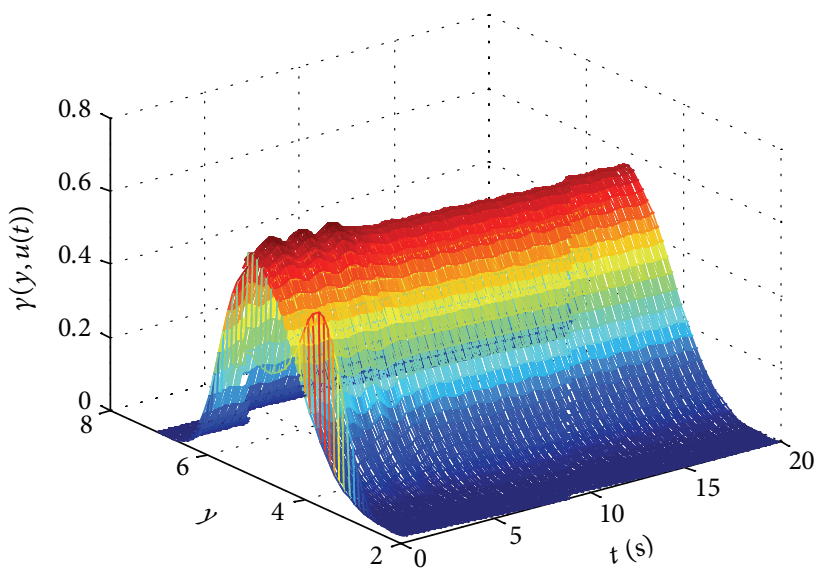

FIGURE 5: The output PDF of the whole process with fault tolerant control.

make the postfault PDF still track the desired PDF. The Lyapunov stability theorem and LMI method are used to analyze the stability of the augmentation observation error dynamic system and tracking error dynamic system, and $H_{\infty}$ performance of fault diagnosis is guaranteed. The simulations have further confirmed the efficiency of the proposed fault diagnosis and fault tolerant control algorithm.

\section{Competing Interests}

The authors declare that they have no competing interests.

\section{Acknowledgments}

This work was supported by the National Natural Science Foundation of China (No. 61374128), State Key Laboratory of Synthetical Automation for Process Industries, the Science and Technology Innovation Talents 14HASTIT040 in Colleges and Universities in Henan Province, China, and Excellent Young Scientist Development Foundation 1421319086 of Zhengzhou University, China. 


\section{References}

[1] E. A. García and P. M. Frank, "Deterministic nonlinear observer-based approaches to fault diagnosis: a survey," Control Engineering Practice, vol. 5, no. 5, pp. 663-670, 1997.

[2] J. Chen and R. J. Pantton, Robust Model-Based Fault Diagnosis for Dynamic Systems, Kluwer Academic, Boston, Mass, USA, 1999.

[3] R. Isermann, "Model-based fault-detection and diagnosisstatus and applications," Annual Reviews in Control, vol. 29, no. 1, pp. 71-85, 2005.

[4] E. Martin, J. Morris, and S. Lane, "Monitoring process manufacturing performance," IEEE Control Systems Magazine, vol. 22, no. 5, pp. 26-39, 2002.

[5] H. Wang, Bounded Dynamic Stochastic Systems, Advances in Industrial Control, Springer, London, UK, 2000.

[6] L. Guo and H. Wang, "Fault detection and diagnosis for general stochastic systems using B-spline expansions and nonlinear filters," IEEE Transactions on Circuits and Systems I: Regular Papers, vol. 52, no. 8, pp. 1644-1652, 2005.

[7] L. Yao, J. Qin, A. Wang, and H. Wang, "Fault diagnosis and faulttolerant control for non-Gaussian non-linear stochastic systems using a rational square-root approximation model," IET Control Theory \& Applications, vol. 7, no. 1, pp. 116-124, 2013.

[8] L. N. Yao, J. F. Qin, H. Wang, and B. Jiang, "Design of new fault diagnosis and fault tolerant control scheme for non-Gaussian singular stochastic distribution systems," Automatica, vol. 48, no. 9, pp. 2305-2313, 2012.

[9] L. Guo and Y. Zhang, "Generalized robust $H_{\infty}$ fault diagnosis filtering based on conditional stochastic distributions of system outputs," Proceedings of the Institution of Mechanical Engineers. Part I: Journal of Systems \& Control Engineering, vol. 221, no. 6, pp. 857-864, 2007.

[10] L. Yao, Z. Yin, and H. Wang, "Robust $H_{\infty}$ fault diagnosis for stochastic distribution systems with disturbance rejection performance," International Journal of Modelling, Identification and Control, vol. 21, no. 3, pp. 288-294, 2014.

[11] Q. K. Shen, B. Jiang, and V. Cocquempot, "Fault-tolerant control for T-S fuzzy systems with application to near-space hypersonic vehicle with actuator faults," IEEE Transactions on Fuzzy Systems, vol. 20, no. 4, pp. 652-665, 2012.

[12] L. N. Yao and B. Peng, "Fault diagnosis and fault tolerant control for the non-Gaussian time-delayed stochastic distribution control system," Journal of the Franklin Institute. Engineering and Applied Mathematics, vol. 351, no. 3, pp. 1577-1595, 2014.

[13] L. Yao and L. Feng, "Fault diagnosis and fault tolerant control for non-Gaussian time-delayed singular stochastic distribution systems," International Journal of Control, Automation and Systems, vol. 14, no. 2, pp. 235-442, 2016.

[14] M. Liu, X. Cao, and P. Shi, "Fault estimation and tolerant control for fuzzy stochastic systems," IEEE Transactions on Fuzzy Systems, vol. 21, no. 2, pp. 221-229, 2013.

[15] H. Wang, "Minimum entropy control of non-Gaussian dynamic stochastic systems," IEEE Transactions on Automatic Control, vol. 47, no. 2, pp. 398-403, 2002.

[16] L. Yao, Y. Guan, and A. Wang, "Fault diagnosis and minimum entropy fault tolerant control for non-Gaussian singular stochastic distribution systems using square-root approximation," International Journal of Modelling, Identification and Control, vol. 24, no. 3, pp. 206-215, 2015.
[17] J. Zhou, G. Li, and H. Wang, "Robust tracking controller design for non-Gaussian singular uncertainty stochastic distribution systems," Automatica, vol. 50, no. 4, pp. 1296-1303, 2014.

[18] L. N. Yao and L. Feng, "Fault diagnosis and fault tolerant tracking control for the non-Gaussian singular time-delayed stochastic distribution system with PDF approximation error," Neurocomputing, vol. 175, pp. 538-543, 2016.

[19] L.-N. Yao and H. Wang, "Fault diagnosis and tolerant control for non-Gaussian stochastic distribution control systems based on the rational square-root approximation model," Control Theory and Applications, vol. 23, no. 4, pp. 561-568, 2006.

[20] S. Cao, Y. Yi, and L. Guo, "Anti-disturbance fault diagnosis for non-Gaussian stochastic distribution systems with multiple disturbances," Neurocomputing, vol. 136, pp. 315-320, 2014.

[21] L. Guo and W.-H. Chen, "Disturbance attenuation and rejection for systems with nonlinearity via DOBC approach," International Journal of Robust and Nonlinear Control, vol. 15, no. 3, pp. 109-125, 2005. 


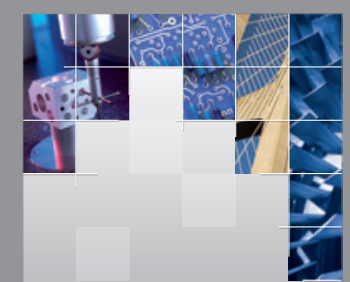

\section{Enfincering}
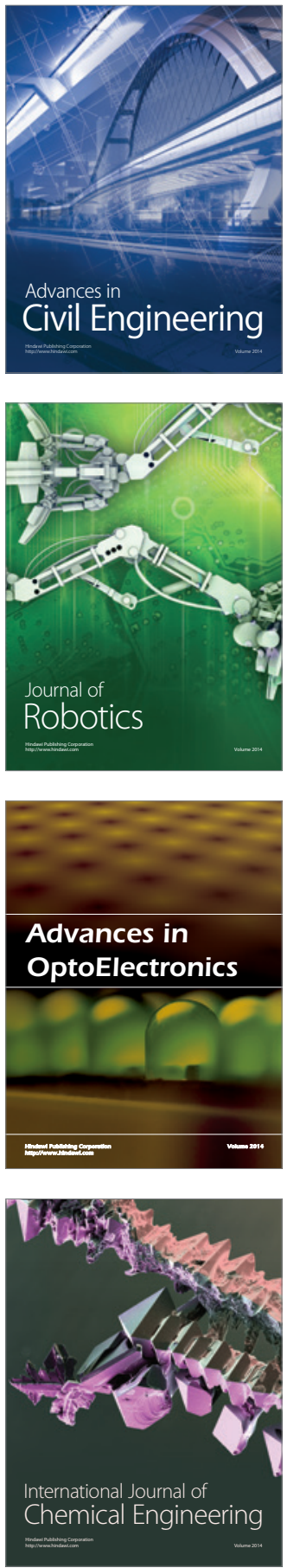

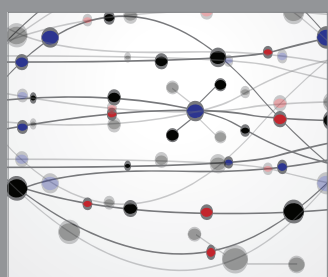

The Scientific World Journal

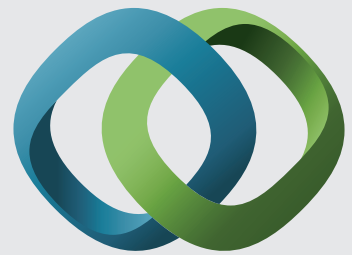

\section{Hindawi}

Submit your manuscripts at

http://www.hindawi.com
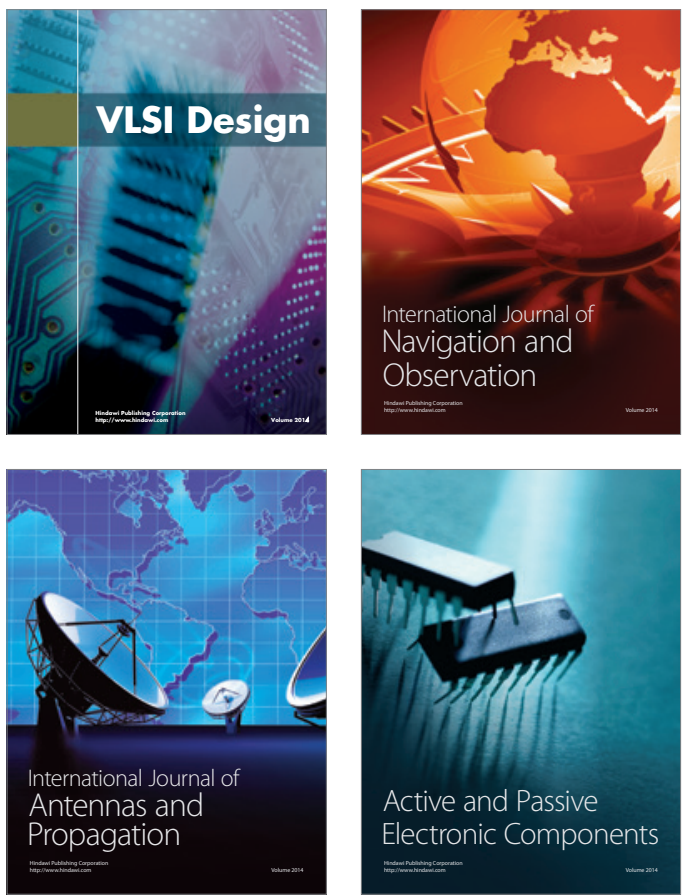
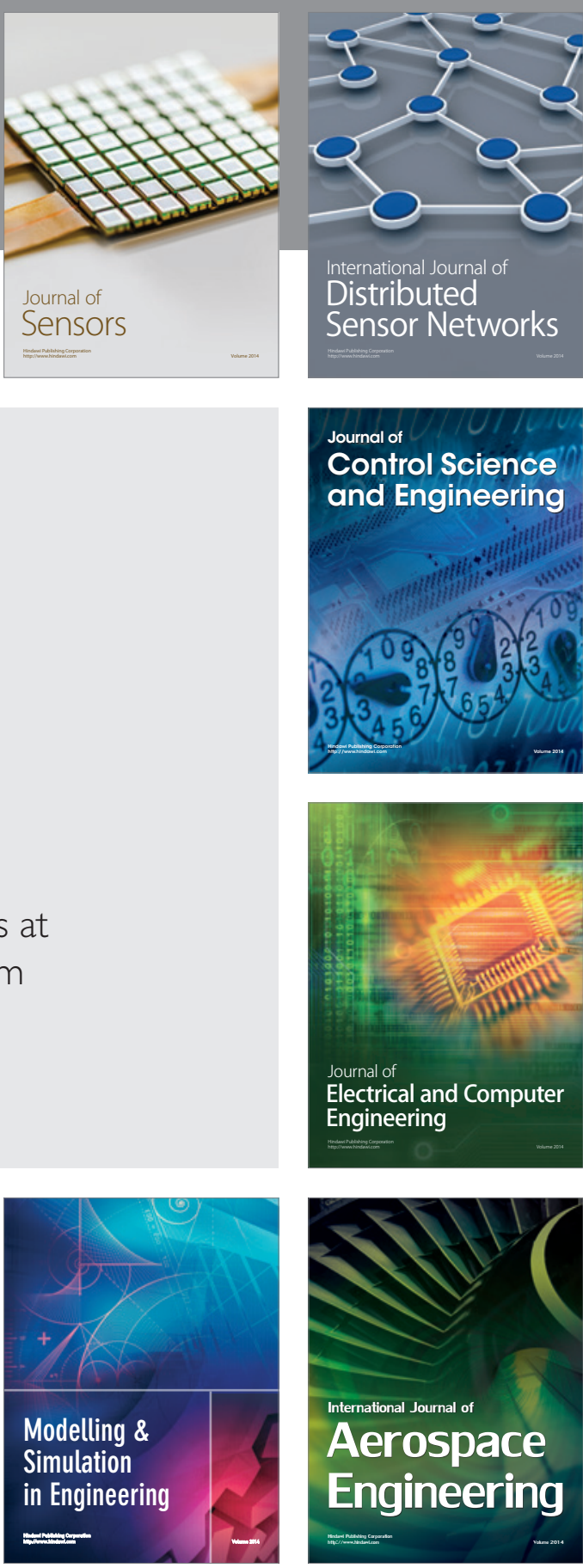

International Journal of

Distributed

Sensor Networks

Journal of

Control Science

and Engineering
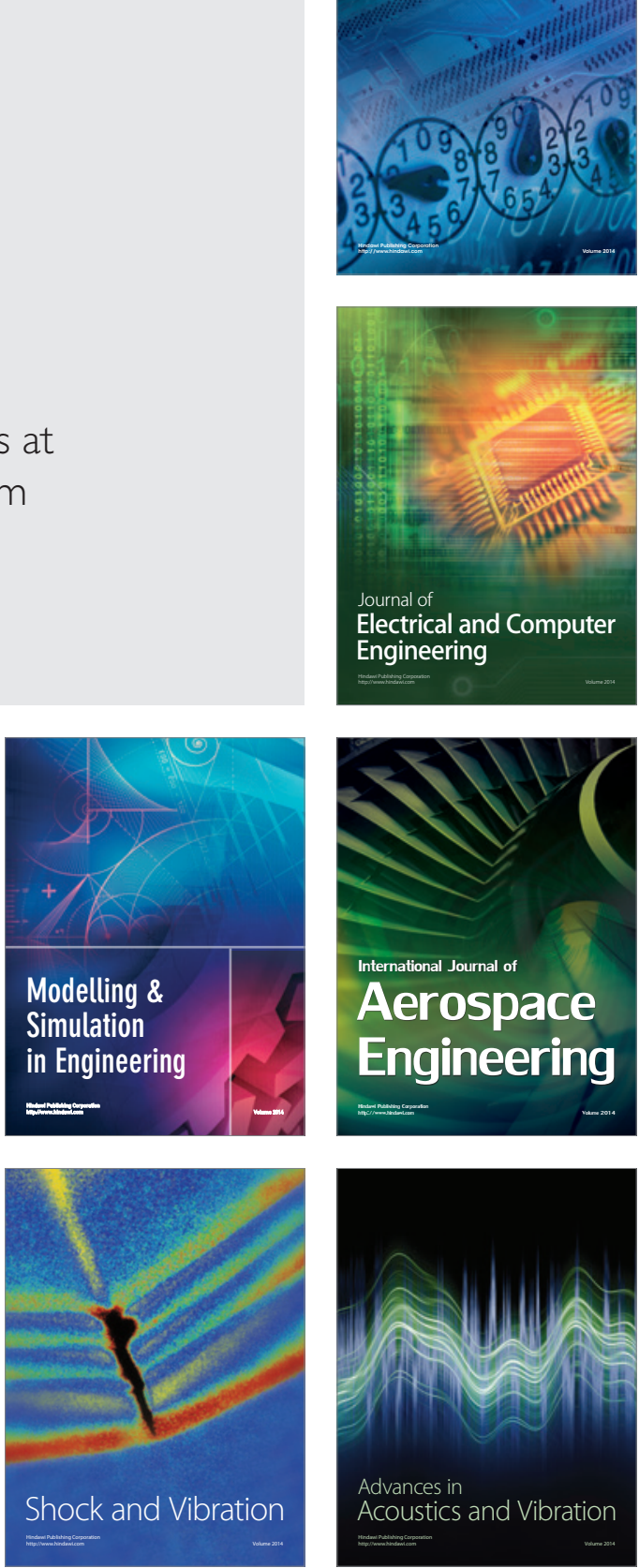